\title{
Budgethilfe und die Deklaration von Paris: „Eine Frage des Massstabs" Fallbeispiel Mali
}

\author{
Jean-Luc Virchaux
}

\section{(2) OpenEdition}

\section{Journals}

Electronic version

URL: http://journals.openedition.org/sjep/410

DOI: $10.4000 /$ sjep.410

ISSN: 1663-9677

\section{Publisher}

Institut de hautes études internationales et du développement

\section{Printed version}

Date of publication: 1 novembre 2007

Number of pages: $227-234$

ISBN: 978-2-88247-069-0

ISSN: 1660-5926

Electronic reference

Jean-Luc Virchaux, «Budgethilfe und die Deklaration von Paris: „Eine Frage des Massstabs“

Fallbeispiel Mali », Schweizerisches Jahrbuch für Entwicklungspolitik [Online], 26-2 | 2007, Online erschienen am: 03 Juni 2010, abgerufen am 07 September 2020. URL : http:// journals.openedition.org/sjep/410; DOI : https://doi.org/10.4000/sjep.410 


\title{
Budgethilfe und die Deklaration von Paris: „Eine Frage des Massstabs" Fallbeispiel Mali
}

\author{
Jean-Luc Virchaux*
}

D er engagierte Beobachter Westafrikas kommt heute nicht umhin, sich mit den Millenniumsentwicklungszielen (MDG), den Strategienrahmen zur Armutsbekämpfung (Poverty Reduction Strategy Papers - PRSP), der Budgethilfe und den „hochrangigen“ Erklärungen, namentlich der Deklaration von Paris über die Wirksamkeit der Hilfe, auseinanderzusetzen.

Die folgenden Ausführungen zielen nicht darauf ab, gegen die neuen Vorhaben in der internationalen Entwicklungszusammenarbeit zu polemisieren. Vielmehr sollen sie anhand von zwei Fallbeispielen die Wirksamkeit (um den mittlerweile unverzichtbaren Jargon der Entwicklungszusammenarbeiter aufzugreifen) dieser Massnahmen aufzeigen. Als Beispiele dienen das Baumwollanbaugebiet im südlichen Mali und die Landeshauptstadt Bamako. Die Analyse der beiden Fallbeispiele erfolgt aus zwei unterschiedlichen Perspektiven: Im Süden des Landes, wo greifbare Ergebnisse der neuen Entwicklungshilfestrategien noch ausstehen, wird eine mikroökonomische Sichtweise angelegt, in der Hauptstadt, die mit Stolz ermutigende Ergebnisse aufweisen kann, eine makroökonomische. Beide Szenarien sind von dem politischen Willen vormaliger Regierungen geprägt, die politische Macht im Lande schrittweise zu dezentralisieren. Als Antwort auf die wiederholten Aufstände nordmalischer Bevölkerungsgruppen hatte die erste demokratische Regierung des Landes im Jahr 1992 Überlegungen zur politischen Neuorganisation des Landes eingeleitet, die die Übertragung zahlreicher Kompetenzen an künftige lokale politische Kräfte vorsah. Nach einem langwierigen Konkretisierungs- und Koordinierungsprozess wurde das Konzept der Dezentralisierung, das die Einführung der drei Gebietskörperschaften Region, Kreis und Kommune vorsieht, in den ersten Gemeindewahlen im Jahr 1999 bestätigt.

Vor der Analyse der beiden Fallbeispiele soll darauf hingewiesen werden, dass die neuen Strategien keine Alternativen zu älteren Ansätzen der Entwicklungszusammenarbeit darstellen, sondern vielmehr zu einem festen Bestandteil der Entwicklungslandschaft geworden sind und diese über zahlreiche Jahre hinweg prägen werden. ${ }^{1}$ Aber es ist an der Zeit, darüber nachzudenken, wie sie verbessert oder neu ausgerichtet werden können. In diesem Zusammenhang sind die Erfahrungen der schweizerischen Entwicklungszusammenarbeit in Mali insofern interessant, als sie einige neue Denkansätze aufzeigen. Die nachfolgenden Ausführungen zielen darauf ab, diese Ansätze kurz zu skizzieren.

\footnotetext{
Leiter des Schweizerischen Koordinationsbüro Mali, Bamako, Mali.

Nicht zuletzt deshalb, weil die Zuwendungsbeträge erheblich sind und nur Zentralregierungen - und auch diese zuweilen nur mit grosser Mühe - in der Lage sind, die Beträge zu absorbieren. Die öffentliche Entwicklungshilfe für Mali (ÖEH) hat in den letzten Jahren tendenziell zugenommen. Im Jahr 2006 machte sie rund 10,3\% des malischen Bruttoinlandprodukts (BIP) aus. Die globale und sektorielle Budgethilfe stellt $40 \%$ des gesamten Staatshaushalts. 15\% der Budgethilfe fliessen in den Betriebshaushalt, $70 \%$ in das Investitionsbudget.
} 


\section{Koutiala: Stimmt der Eindruck?}

Koutiala, Verwaltungssitz des gleichnamigen Kreises ${ }^{2}$, ist eine in der dritten Region Malis gelegene mittelgrosse Stadt mit über 100000 Einwohnern. Ihre wirtschaftliche Bedeutung reicht über den Kreis hinaus und erstreckt sich auf 45 ländliche Gemeinden mit über 600000 Einwohnern. Koutiala gilt als Hauptstadt der Baumwolle, des wichtigsten Exportprodukts der malischen Wirtschaft. Im Laufe der letzten 30 Jahre wurde dort eine Reihe von Betrieben für die Weiterverarbeitung der Baumwolle aufgebaut ${ }^{3}$. Dank der privilegierten Lage als wirtschaftliches Zentrum gilt Koutiala als einer der wohlhabendsten Kreise des Landes. Aber trifft dies auch wirklich zu?

Seit 2005 streben die Gemeinde und die politischen Behörden des Kreises eine bessere Nutzung des wirtschaftlichen Potenzials der Stadt und ihres Einflussbereichs an. Zu diesem Zwecke lancierten sie mit Unterstützung der Direktion für Entwicklung und Zusammenarbeit (DEZA) eine sogenannte ECOLOC-Studie ${ }^{4}$, die ein Abbild der lokalen Wirtschaft zeichnen sollte. Von dieser Bestandsaufnahme ausgehend sollten in Zusammenarbeit mit allen am Entwicklungsprozess beteiligten Akteuren die wirtschaftlichen Potenziale der Stadt Koutiala und ihres Hinterlandes identifiziert und die Wirtschaftspolitik des Kreises und der Gemeinden für die kommenden 15 Jahre definiert werden. Ein malisches Fachbüro führte die Studie über einen Zeitraum von 18 Monaten durch. Zwanzig Befrager identifizierten die beteiligten Wirtschaftsakteure und erfassten die Art und den Umfang ihrer Tätigkeiten sowie die Finanzflüsse innerhalb des Gebietes.

Im März 2006 wurden die ersten Ergebnisse einem breiten Publikum, lokalen Handlungsträgern und Vertretern zahlreicher Ministerien und Entwicklungshilfeorganisationen vorgestellt ${ }^{5}$. Zur allgemeinen Überraschung ergab sich ein völlig unerwartetes Bild: Im Allgemeinen war davon ausgegangen worden, dass der Wohlstand des Gebiets weit über dem Landesdurchschnitt liegt; mit einem Betrag von 115000 FCFA pro Jahr und Kopf (rund 300 Schweizer Franken) war das lokale BIP pro Jahr und Kopf jedoch nur halb so hoch wie der entsprechende Wert für den Rest des Landes. Diese Erkenntnis stellte die Welt der lokalen Behördenvertreter im Mikrokosmos Minyanka auf den Kopf: Hatten sie sich bislang für die Reichen in einem Land der Armen gehalten, so mussten sie nun einsehen, dass sie zu den Armen unter den Armen zählten. Wirtschaftsexperten und Statistiker meldeten vehemente Zweifel an der Untersuchung an und behaupteten unter anderem, die Methode sei falsch. „Das mag sein“, räumte der

2 Politisch-administrativ ist Mali in acht Regionen, 49 Kreise und 703 Kommunen und den HauptstadtDistrikt Bamako gegliedert. Koutiala zählt zur dritten Region mit Sikasso als Hauptstadt.

3 Mit ihren acht Industrieanlagen (Ölmühlen, Egrenieranlagen und Futtermühlen) ist Koutiala nach Bamako die zweitwichtigste Industriestadt Malis.

4 Die ECOLOC-Methode (ECOLOC steht für Aufschwung der lokalen Wirtschaft) wurde vom SahelClub der OECD entwickelt. Die breite Anwendung der Methode wird von dem in Cotonou ansässigen Partenariat pour le Développement Municipal (PDM) gewährleistet. Die Gesamtheit der rund 20 ECOLOC-Studien, einschliesslich jener in Koutiala, wird vom PDM regelmässig betreut.

5 Die sieben thematischen Studien sowie eine Zusammenfassung sind bei der Kommunalverwaltung von Koutiala sowie im Schweizerischen Kooperationsbüro in Bamako erhältlich. Die Ergebnisse werden Ende 2007 auf der Website der Kommunalverwaltung veröffentlicht. 
Leiter der Studie ein, ,aber vor der Untersuchung hatten wir keinerlei Zahlen, nun haben wir wenigstens einen Anhaltspunkt ${ }^{6}$." In der Tat war dies das erste Mal, dass in Mali eine ECOLOC-Studie abgeschlossen und die lokale Wirtschaftslage evaluiert wurde. Die Studie wurde beim Africities Summit 2006 in Nairobi in Anwesenheit zahlreicher Entwicklungsexperten vorgestellt ${ }^{7}$, ohne dass ihre Methode oder Resultate in Frage gestellt wurden. Dies darf als Beweis für die Seriosität der Untersuchung und die Aussagekraft ihrer Ergebnisse interpretiert werden, die in erster Linie für die politischen Entscheidungsträger, die Wirtschaftsakteure und die lokalen Planer von Interesse sind.

Ein weiteres Ergebnis der Studie regt ebenfalls zum Nachdenken an, nämlich das bestehende Ungleichgewicht zwischen dem Zentralstaat und einer Randzone wie Koutiala. Der Anteil der vom Staatshaushalt bereitgestellten Finanzmittel macht weniger als 2\% des lokalen BIP aus. Da das Staatsbudget seinerseits rund 30\% des nationalen BIP darstellt, muss der Schluss gezogen werden, dass die Wirtschaft Koutialas in nur äusserst geringem Masse vom Staatshaushalt profitiert. Diese Einsicht mag zwar nicht überraschen, aber die Tatsache, dass sie nun durch konkrete Zahlen untermauert werden kann, veranschaulicht, wie tief die Kluft zwischen Zentrum und Peripherie in Wirklichkeit ist. Die schiere Existenz dieser Kluft stellt zudem die aktuelle Ausrichtung der Entwicklungszusammenarbeit in Frage, einer Zusammenarbeit, die auf globale und sektorielle Budgethilfe setzt und aus Gründen der Wirksamkeit die Unterstützung eines, wie oben dargestellt, zentralisierten Staatshaushalts begünstigt.

\section{Sikasso : dezentralisierte Budgethilfe - Häresie oder Notwendigkeit?}

Die im Jahr 1999 als Gebietskörperschaft eingerichtete Region Sikasso hat eine Fläche von $75000 \mathrm{~km}^{2}$ und rund zwei Millionen Einwohner. Obwohl dort dank ausreichender Niederschlagsmengen Landwirtschaft (Baumwolle, Getreide, Gemüse und Obst) und Viehhaltung betrieben werden können und die Region damit ein grösseres wirtschaftliches Potenzial als andere Teile des Landes besitzt, ist sie ärmer als der Landesdurchschnitt ${ }^{8}$. Als Folge des im Juni 2002 getroffenen Entscheids, Kompetenzen des Staates an die Gebietskörperschaften zu übertragen, hat das Regionalparlament heute zahlreiche Aufgaben in den Bereichen Bildung, Gesundheit und soziale und wirtschaftliche Entwicklung inne9. Sein Haushalt wird weitgehend über einen Anteil der Steuern auf Regional- und Lokalentwicklung in Höhe von $10 \%$ gespeist, die auf Kommunalebene erhoben werden ${ }^{10}$. Im Jahr 2005 betrug dieses Budget rund 200 Millionen FCFA (rund 500000 Schweizer Franken). Ein derart bescheidener Haushalt verpflichtet ein

\footnotetext{
Zitat Massa Coulibaly, Wirtschaftsstatistiker und Leiter der ECOLOC-Studie. Abdel Kader Dicko, Sigida Koura, Koutiala pour un nouveau cadre de vie, Oktober 2006.

$70 \%$ der Bevölkerung leben unterhalb der Armutsgrenze und haben eingeschränkten Zugang zu Bildung, Gesundheitsversorgung, Trinkwasser usw.

9 Die Zuständigkeiten hängen von der Hierarchiestufe der Gebietskörperschaft ab. Im Bildungsbereich beispielsweise ist die Kommune zuständig für die Grundbildung (Grundschule) und die nichtschulische Bildung, der Kreis für die Mittelstufe und die Region für die Oberschule und die berufliche Bildung. Die jeweiligen Hierarchiestufen der Gebietskörperschaften haben keine Regelungskompetenz für die anderen Ebenen.

10 Pro-Kopf-Steuer zwischen 1000 und 2000 FCFA, von der 60\% der Kommune, 30\% dem Kreis und $10 \%$ dem Regionalparlament zufliessen.
} 
Regionalparlament, bei der Definition der politischen Strategien klare Prioritäten zu setzen.

Im Jahr 2002 bat das Regionalparlament die DEZA um Unterstützung beim Kapazitätsaufbau mit dem Ziel, die Wahrnehmung der neu übertragenen Kompetenzen sicherzustellen. In einem ersten Schritt wurde eine sozioökonomische Untersuchung der Region erstellt, um den Grad der Armut und die wesentlichen wirtschaftlichen Potenziale der Region zu identifizieren. Die strategische Wahl der Abgeordneten fiel rasch auf die Erarbeitung einer Wirtschaftsförderungspolitik, denn bei ,einem Armutsgrad von $70 \%$ ist nicht die Armut an sich das Problem, sondern der Entwicklungsrückstand"11. Gemeinsam mit Vertretern von Fachorganisationen und Wirtschaft reisten Abgeordnete in Nachbarländer sowie in die Schweiz und nach Frankreich, um vor Ort in Erfahrung zu bringen, auf welche Weise Gebietskörperschaften die lokale Wirtschaft fördern können, welchen Handlungsspielraum sie haben und welche Instrumente ihnen zur Verfügung stehen. Auf der Grundlage der gewonnenen Erkenntnisse definierte das Regionalparlament eine Strategie für die ländliche Entwicklung, die sich auf neun land- und viehwirtschaftliche Bereiche konzentriert. Mit den in diesen Bereichen tätigen Handlungsträgern wurden Aktionspläne ausgearbeitet und durch Investitionsprogramme abgestützt, die auf eine Verbesserung der Rahmenbedingungen abzielen ${ }^{12}$.

Angesichts der Tatsache, dass im Jahr 2005 75\% des Staatshaushalts der Landeshauptstadt, $24 \%$ den dezentralisierten staatlichen Institutionen und nur knapp als 1\% den Gebietskörperschaften zuflossen, blieb das dringende Finanzierungsproblem des Regionalparlaments weiterhin ungelöst ${ }^{13}$. Welche Finanzierungsstrategie sollte gewählt werden? Wie sollten lokale Finanzmittel mobilisiert werden? Wie sollte der Zugang zu den in Bamako verfügbaren Mitteln gewährleistet werden, namentlich zu jenen der nationalen, von den technischen Ministerien verwalteten Sektoralprogramme? Und wie sollte es gelingen, die Finanzflüsse an die dezentralisierten unteren Ebenen zu leiten?

In einem ersten Schritt verhandelte das Regionalparlament mit den Gebietskörperschaften der unteren Hierarchiestufen (Kreise und Gemeinden) über ihre Beteiligung an der Finanzierung von Infrastrukturmassnahmen in ihren jeweiligen Gebieten. In einem zweiten Schritt wählte es mit Unterstützung der DEZA den Weg der Budgethilfe. Diese Entscheidung führte dazu, dass DEZA-Beiträge im Haushalt 2007 eingestellt wurden ${ }^{14}$. Die Strategie zeigte Erfolg und motivierte neue Geber wie beispielsweise die Agence Française de Développement, sich ebenfalls an dem Vorhaben zu beteiligen. Als Ergebnis dieses Vorgehens konnte das Budget des Regionalparlaments im Jahr 2007 auf 2,7 Milliarden

11 Zitat eines Abgeordneten des Regionalparlaments.

12 Sanierung der Zugangswege zu Produktionszonen, Aufbau regionaler Infrastrukturen zur Verarbeitung und Lagerung von Erzeugnissen, Aufbau von berufsübergreifenden Strukturen für die Planung von Produktion und Vertrieb, Konzentration auf die Versorgung nationaler Märkte, Gewährleistung des Austauschs usw.

13 Sekretariat der PRSP, Evaluationsbericht zur Umsetzung der PRSP im Jahr 2005, April 2006.

14 Die DEZA gewährt Mali keine allgemeine oder sektorielle Budgethilfe auf nationaler Ebene, sie arbeitet aber derzeit an einem Pilotprozess, der eine Logik der Budgethilfe auf lokaler Ebene (Regionalversammlung von Sikasso) vorsieht. 
FCFA (rund 7 Millionen Schweizer Franken) erhöht werden, ohne dass die Mittelflüsse aus dem Staatshaushalt stiegen. Dieses zweite Beispiel zeigt, dass das Problem einer gerechten Aufteilung eines durch Budget- und Sektorenhilfe subventionierten Staatshaushalts bestehen bleibt und auf lokaler Ebene von Gebern alleine nicht gelöst werden kann und auch nicht gelöst werden sollte.

\section{Budgethilfe: ein Blick von oben}

„In Mali gehts aufwärts!“ Mali ist 33-mal grösser und hat rund doppelt so viele Einwohner wie die Schweiz. In den letzten 15 Jahren ist es dem Land gelungen, ein demokratisches System mit anerkannten Institutionen ${ }^{15}$ aufzubauen und einen Dezentralisierungsprozess einzuleiten. Letzterer hat seit 1999 mit den Wahlen in der Hauptstadt, den acht Regionen, 49 Kreisen und 703 Gemeinden konkretere Züge angenommen. Präsidentschafts-, Parlaments- und Kommunalwahlen finden regelmässig und unter relativ transparenten Bedingungen statt. Obwohl das Land den vorletzten Platz auf der Liste der ärmsten Länder belegt, ${ }^{16}$ hat es in den letzten zehn Jahren ein durchschnittliches Wirtschaftswachstum von $5 \%$ erzielt und angesichts der Wahrung des makroökonomischen Gleichgewichts die Anerkennung des IWF gewonnen. In der Landwirtschaft kommen vorwiegend traditionelle Anbaumethoden zum Zuge, aber die Produktionserträge steigen und decken den Getreidebedarf der Landesbevölkerung, die eine Wachstumsrate zwischen 2,7 und 3\% verzeichnet. Die Baumwollproduktion befindet sich in einer Krise und verliert an Wettbewerbsfähigkeit. Letzteres ist auf die Schwierigkeiten des Staates bei der Verbesserung der nationalen Rahmenbedingungen sowie auf die sinkenden Weltmarktpreise zurückzuführen, die ihrerseits durch massive Subventionen anderer Baumwollexportländer - beispielsweise der Vereinigten Staaten - bedingt sind. Die Einbussen bei der Baumwollproduktion werden indessen teilweise durch eine Erhöhung der Goldproduktion ausgeglichen ${ }^{17}$. Testbohrungen weisen auf wirtschaftlich nutzbare Gas- und Erdölvorkommen hin.

Unter Berücksichtigung der Tatsache, dass der gesamte malische Staatshaushalt ${ }^{18}$ nur gerade einem Drittel des Haushalts des Kantons Genf bzw. dem Doppelten des Haushalts der Stadt Genf entspricht, sind die Anstrengungen Malis namentlich unter dem Blickwinkel einer Kosten-Nutzen-Analyse beachtlich. Die guten Leistungen des Landes zahlen sich aus: Seit der Dezentralisierung sind die Entwicklungshilfegelder stetig gestiegen und betragen nun rund 600 Millionen USD pro Jahr ${ }^{19}$, was ungefähr 11,7\% des BIP entspricht. Angesichts der breit gestreuten Hilfemassnahmen, die häufig den Prozessen im Lande widersprechen und die politischen Strategien und staatlichen Kapazitäten nur unzureichend stärken, haben sich einige Geber $^{20}$ in den frühen 2000er-Jahren

15 Parlament, Gemeinderäte, Gebietskörperschaften.

16 Rang 175 von 176 bewerteten Ländern.

17 Mali ist nach Südafrika und Ghana der drittwichtigste Goldproduzent Afrikas.

18 Der Staatshaushalt liegt bei knapp 2 Milliarden Schweizer Franken.

19 Zum Vergleich: 1,05 Milliarden USD für den Senegal (13,5\% des BIP), 1,75 Milliarden USD für Tansania (16,1\% des BIP). Quelle: United Nations Development Programme (UNDP), Human Development Report 2006, Paris, Economica, 2006.

20 Europäische Union, Frankreich, Niederlande. 
entschieden, der malischen Regierung Budgethilfe zu leisten. Dieser neue Ansatz hat es der Regierung unter anderem erleichtert, einen ersten Strategierahmen zur Armutsbekämpfung (PRSP 2002-2006) zu definieren. Dieser Strategierahmen ist zwar bei weitem nicht perfekt, dient aber der Regierung als Anhaltspunkt und unterstützt die Harmonisierung der strategischen Ausrichtungen der Geber. Die Deklaration von Paris über die Wirksamkeit der Hilfe von 2005, die von der Mehrzahl der OECD-Länder und auch von der Schweiz unterzeichnet wurde, beschleunigte das Bemühen der Geber, Hilfsgelder für Massnahmen der globalen oder sektoriellen Budgethilfe zu kanalisieren. Im Zuge dieser Dynamik haben sich neun bilaterale und multilaterale Geber verpflichtet, Mali Budgethilfe zu gewähren, und sieben haben bereits 2006 eine Partnerschaft mit der malischen Regierung unterzeichnet.

Die Fortschritte bei der Umsetzung der Deklaration von Paris sind für die Geber offenkundig. Die Harmonisierung der Massnahmen wurde verbessert, im politischen Dialog mit der Regierung zeichnen sich immer häufiger gemeinsame Positionen $\mathrm{ab}$, und die Geber beginnen - namentlich über die Budgethilfe vermittelt sich an den politischen Strategien und Prozessen der Regierung auszurichten. Die malische Regierung hat eine Palette von Reformen an mehreren Fronten eingeleitet. Im Streben nach grösserer Transparenz und höherer Effizienz des Haushalts kommt der Optimierung der Finanz- und Budgetflüsse besondere Bedeutung zu. Die gegenwärtige Reform der Entwicklungshilfe ist für alle Beteiligten äusserst anspruchsvoll, sowohl im Hinblick auf den Zeitaufwand als auch hinsichtlich der für die Umsetzung erforderlichen personellen Ressourcen.

Die Fortschritte bei der Umsetzung der Deklaration von Paris sind unverkennbar. Allerdings muss eingeräumt werden, dass die neue Dynamik bislang im Wesentlichen nur auf der Ebene des Zentrums greift, obwohl die Beteiligung aller Akteure am Entwicklungsprozess ${ }^{21}$ - wenn auch öfter in offiziellen Erklärungen beschworen als tatsächlich umgesetzt - als Vorbedingung für Nachhaltigkeit und Erfolg des Reformprozesses gilt.

Von oben betrachtet zeichnen sich greifbare Fortschritte ab, auch wenn es zum gegenwärtigen Zeitpunkt noch schwierig ist, die Auswirkungen auf die Gesellschaft, die Lebensbedingungen, die Bewältigung der Armut und die Entwicklung zu evaluieren.

\section{Schlussfolgerungen: die Grenzen einer ehrgeizigen Reform von oben}

Die konkreten Erfahrungen in Koutiala und Sikasso veranschaulichen die Spannungen zwischen Zentrum und Peripherie. Die Zentripetalkräfte der Konzentration finanzieller Ressourcen sind bislang stärker als die Zentrifugalkräfte, die eine Verlagerung der Mittelflüsse hin zu den neu entstehenden politischen Institutionen suchen, die die eigentlichen Träger der Entwicklung sind. An diesem Punkt stellt sich die Frage, ob der an die Deklaration von Paris geknüpfte Reformprozess, der eine globale oder sektorielle Budgethilfe begünstigt, längerfristig tatsächlich eine ausgeglichenere Verteilung des Staatshaushalts zugunsten der dezentralisierten Körperschaften fördert.

21 Branchenverbände, Zivilgesellschaft, NGO, Gebietskörperschaften. 
Angesichts des Eifers, mit dem die Regierung den Dezentralisierungsprozess vorantreibt, und angesichts der Qualität des politischen Dialogs zwischen der Regierung und den Gebern sind Fortschritte zu erkennen. Der Staatshaushalt 2007 sieht eine Zunahme der Mittelflüsse zugunsten der dezentralisierten staatlichen Dienste 22 um $740 \%{ }^{23}$ vor. Die Gebietskörperschaften werden selbstverständlich nicht direkt von diesen Zuwendungen profitieren, und die Transfermechanismen sind auch noch nicht klar definiert. So zeichnen sich bereits heute Spannungen zwischen den dezentralisierten staatlichen Diensten und den Gebietskörperschaften in Bezug auf die Verwaltung der Gelder ab.

Der Rhythmus und die Komplexität der an globale oder sektorielle Budgethilfe geknüpften Reformen erfordert ein ausserordentlich hohes Kompetenzniveau der Geber und der malischen Regierung. Die diplomatischen Vertretungen und Kooperationsbüros heuern zahlreiche Experten an, während die Ministerien in Bamako schon heute $75 \%$ der Staatskader auf sich vereinen. Unzählige Planungs-, Koordinations-, Global- und Sektoralmeetings werden der Mehrzahl dieser Experten keine Zeit lassen, sich vor Ort zu begeben, um die Lage aus erster Hand zu beurteilen.

Auch der Strategierahmen für Wachstum und Armutsminderung (Growth and Poverty Reduction Strategy Paper, GPRSP), der die PRSP ablöst, sowie der Grundsatz, dass sich die Entwicklungszusammenarbeit an den staatlichen Vorgaben zu orientieren habe, erfordern eine Ausrichtung an klaren, schlüssigen politischen Strategien und den aus diesen abgeleiteten Aktionsplänen. Letztere können nur von oben nach unten konzipiert werden, ohne den jeweiligen lokalen Kontext und die lokalen politischen Kräfte zu berücksichtigen. Die aktuelle Dynamik des Budgethilfeansatzes ist zweifellos von Zentripetalkräften geprägt. Sie konzentrieren Macht und Ressourcen an der Spitze der Staatspyramide, ohne zu garantieren, dass die Hilfsgelder tatsächlich wirksam eingesetzt werden. Dies umso mehr, als die Logik der aktuellen Budgethilfestrategien dem Staat eine quasi Monopolstellung gegenüber dem Privatsektor, den Branchenverbänden und den NGO einräumt, obwohl er bei weitem nicht der einzige Handlungsträger des Entwicklungsprozesses ist.

Angesichts seiner vor Ort gewonnenen Erfahrung ist der Verfasser der Ansicht, dass die Budgethilfe sich nicht mehr ausschliesslich auf eine Reform des Staatsbetriebs konzentrieren sollte, wie es heute der Fall ist, sondern so zu korrigieren ist, dass andere an den politischen Strategien des Staates beteiligten Handlungsträger - namentlich die dezentralisierten Gebietskörperschaften und die zivilgesellschaftlichen Organisationen - ebenfalls eingebunden werden. Das Streben nach einer besseren Wirksamkeit der Entwicklungszusammenarbeit ist eine Frage des Massstabs und der Dosierung.

22 Lokale technische Dienste in den Bereichen Bildung, Gesundheit, Landwirtschaft, Raumplanung, die gemäss der Dezentralisierungsreform die lokalen Gebietskörperschaften unterstützen und beraten sollen.

23 Von 16,5 Milliarden auf 135 Milliarden CFA. 


\section{Republik Mali: Karte der Verwaltungsgliederung}

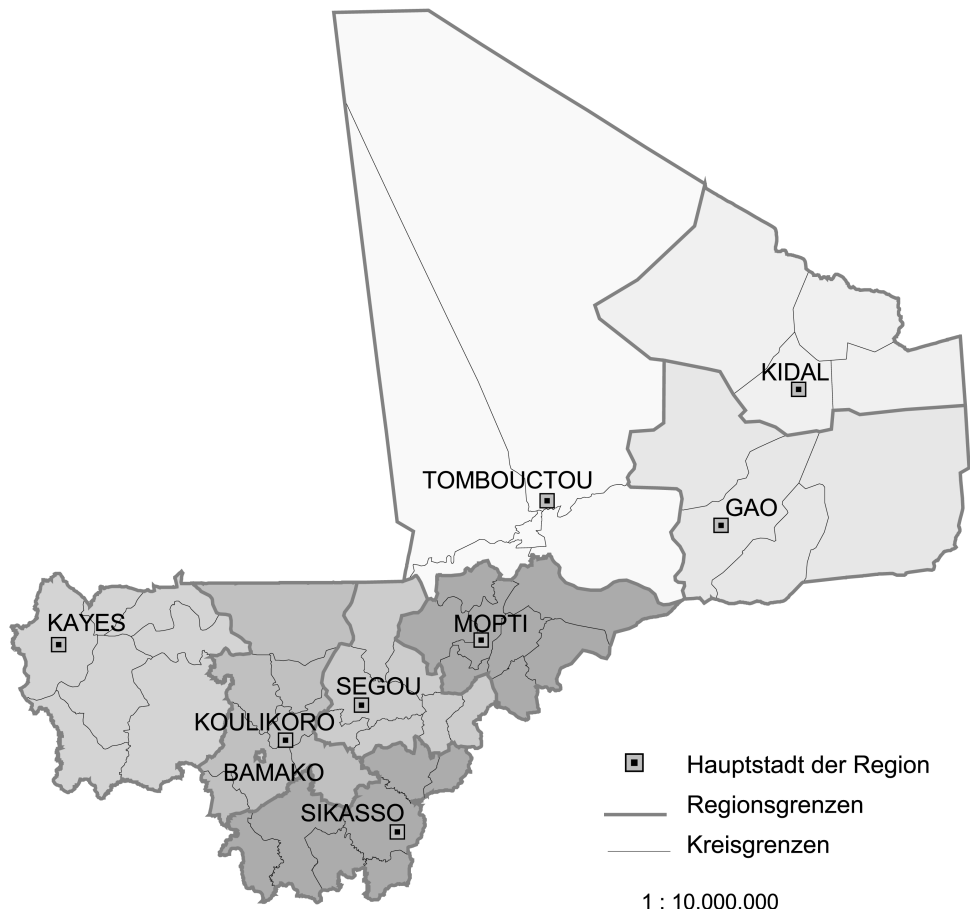

Quelle: Programme d'appui aux collectivités territoriales (PACT), Segou, Mali. 